Urologe 2019.58:1304-1312

https://doi.org/10.1007/s00120-019-01033-7

Online publiziert: 10 . September 2019

(c) Der/die Autor(en) 2019

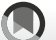

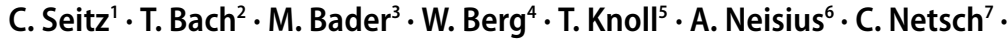
M. Nothacker ${ }^{8} \cdot$ S. Schmidt ${ }^{9} \cdot$ M. Schönthaler ${ }^{10} \cdot$ R. Siener ${ }^{11} \cdot$ R. Stein ${ }^{12} \cdot$ M. Straub ${ }^{13}$. W. Strohmaier ${ }^{14,17} \cdot$ C. Türk ${ }^{15,18} \cdot$ B. Volkmer ${ }^{16}$

'Urologische Universitätsklinik, Medizinische Universität Wien, Allgemeines Krankenhaus der Stadt Wien, Wien, Österreich; ${ }^{2}$ Abteilung für Urologie, Asklepios Klinikum Harburg, Hamburg, Deutschland;

${ }^{3}$ UROClinic München, München, Deutschland; ${ }^{4}$ Urologische Klinik, Uniklinikum Jena, Jena, Deutschland; ${ }^{5}$ Urologische Klinik, Klinikum Sindelfingen-Böblingen, Klinikverbund Südwest, Sindelfingen, Deutschland

${ }^{6}$ Abteilung für Urologie und Kinderurologie, Krankenhaus der Barmherzigen Brüder, Trier, Deutschland;

${ }^{7}$ Abteilung für Urologie, Asklepios Klinik Barmbek, Hamburg, Deutschland; ${ }^{8}$ AWMF-Institut für Medizinisches Wissensmanagement, Berlin, Deutschland; ' ${ }^{1}$ UroEvidence, Deutsche Gesellschaft für Urologie, Berlin, Deutschland; ${ }^{10}$ Klinik für Urologie, Universitätsklinikum Freiburg, Freiburg, Deutschland; "Universitäres Steinzentrum, Klinik und Poliklinik für Urologie und Kinderurologie, Universitätsklinikum Bonn, Bonn, Deutschland; ${ }^{12}$ Zentrum für Kinder-, Jugend- und rekonstruktive Urologie, Universitätsklinikum Mannheim GmbH, Mannheim, Deutschland; ${ }^{13} \mathrm{Klinik}$ und Poliklinik für Urologie, Klinikum rechts der Isar, München, Deutschland; ${ }^{14} \mathrm{Klinik}$ für Urologie und Kinderurologie, RegiomedKlinikum Coburg, Coburg, Deutschland; ${ }^{15}$ Urologische Praxis mit Steinzentrum Wien, Wien, Österreich; ${ }^{16} \mathrm{Klinik}$ für Urologie, Klinikum Kassel, Kassel, Deutschland; ${ }^{17}$ Medical School Regiomed und Akademisches Lehrkrankenhaus, Universität Split, Split, Kroatien; ${ }^{18}$ Urologische Abteilung, Krankenhaus der Barmherzigen Schwestern, Wien, Österreich

\section{Aktualisierung der S2k-Leitlinie zur Diagnostik, Therapie und Metaphylaxe der Urolithiasis (AWMF Registernummer 043- 025)}

\section{Was ist neu?}

\section{Einleitung}

Die erste 2008 von der Deutschen Gesellschaft für Urologie e. V. (DGU) herausgegebene S2k-Leitlinie diente, ebenso wie die jährlich aktualisierte Leitlinie der Europäischen Gesellschaft für Urologie (European Association of Urology, EAU) und die Leitlinien der Amerikanischen Gesellschaft für Urologie (American Urological Association, AUA), als Basis für diese Leitlinie. Eine systematische Literatursuche fand für die vorliegende Aktualisierung im Suchzeitraum Januar 2015 bis Dezember 2017 statt. Zu einigen Themenbereichen wurde selektiv Literatur aus 2018 hinzugefügt. Eine erste überarbeitete Version wurde durch die Steuergruppe erstellt und anschließend von den Arbeitskreismitgliedern Harnsteine ergänzt. Alle Empfehlungen und
Statements wurden online durch alle Leitlinienmitglieder konsentiert, wobei zu jedem der über 130 Empfehlungs- und Statementvorschlägen ein Konsens von zumindest $75 \%$ Zustimmung der Teilnehmer erreicht wurde.

Veränderungen betreffen v. a. die Kapitel: konservative Therapie, Harnsteine bei Kindern, metabolische Diagnostik und Metaphylaxe.

Die konsentierte, auf verfügbarer Evidenz basierende Leitlinie zur Diagnostik, Therapie und Metaphylaxe der Urolithiasis dient der verbesserten Behandlung von Harnsteinerkrankungen. Ärzte und Patienten mit Harnsteinen sollen durch die Leitlinie bei der Entscheidung über Diagnostik, Therapie- und Präventionsmaßnahmen unterstützt werden. Die Leitlinie wurde von der DGU finanziert.
Eine inhaltliche Beeinflussung erfolgte dabei nicht.

Die komplette Fassung der „S2k-Leitlinie zur Diagnostik, Therapie und Metaphylaxe der Urolithiasis“ einschließlich der ausführlichen Beschreibung der Methodik und Referenzen ist auf der Webseite der Arbeitsgemeinschaft der Wissenschaftlichen Medizinischen Fachgesellschaften e. V. (AWMF) unter der Registernummer 043-025 veröffentlicht.

\section{Diagnostik und Bildgebung}

\section{Methoden und Zielsetzung}

Harnsteinbildner weisen aufgrund wiederholt durchgeführter Bildgebungen ein erhöhtes Strahlenexpositionsrisiko auf, wobei die tatsächliche Höhe unbekannt ist [1]. Zwar ist die durchschnittliche 
Strahlenbelastung durch die Computertomographie (CT) in der Harnsteindiagnostik über die Jahre gesunken, aber dennoch höher als nach dem ALARAPrinzip „as low as reasonably achievable" wünschenswert [2]. So können gerade junge Rezidivsteinbildner, die wiederholten CT-Untersuchungen unterzogen werden, erheblichen Effektivdosen ausgesetzt sein [3].

\section{Statement geprüft}

Wer eine bildgebende Diagnostik mit ionisierenden Strahlen plant oder ausübt, ist verpflichtet, jede Strahlenexposition oder Kontamination von Mensch und Umwelt unter Beachtung des Standes von Wissenschaft und Technik und unter Berücksichtigung aller Umstände des Einzelfalls auch unterhalb der Grenzwerte so gering wie möglich zu halten.

Gesamtabstimmung: $100 \%$

\section{Ultraschall}

Der Ultraschall (US) stellt sowohl in der Notfall- als auch in der Routinediagnostik die erste Wahl dar [4]. Die sonographische Darstellung des Harntrakts ist sicher, kann den Aufenthalt in der Notaufnahme signifikant verkürzen [5] und führt zudem zu keinem relevanten Zeitverlust bis zu einer eventuellen Intervention [6, 7]. Der Farbdopplerultraschall eignet sich in der akuten Kolik für die initiale Diagnose eines Harnleitersteins und ist mit den Ergebnissen des Steinsuch-CT vergleichbar [8,9]. Der US führt zwar zu einer Überschätzung der Steingröße [10, 11] aber über spezielle US-Einstellungen (S-Mode) und die Messung der Schallschattenbreite ist eine genauere, mit dem nativen CT vergleichbare Größenbestimmung möglich $[12,13]$.

\section{Empfehlung geprüft}

Der Ultraschall soll die bildgebende Diagnostik der ersten Wahl sowohl in der Akutsituation als auch in der allgemeinen Diagnostik und Nachsorge sein. Gesamtabstimmung: $100 \%$
Hier steht eine Anzeige.

Springer 


\section{Konventionelles Röntgen}

Die Sensitivität der Abdomenübersichtsaufnahme beträgt $44-77 \%$ und die Spezifität 80-87\%. Die Strahlendosis beträgt ca. $0,5 \mathrm{mSv}$. Sie ist hilfreich bei schattengebenden Konkrementen und im Rahmen der Therapiekontrolle/Nachsorge schattengebender Konkremente. Sie ist bei Kindern entbehrlich.

\section{Statement geändert}

Die Röntgenaufnahme der Niere, Harnleiter, Blase und Prostata (Harntraktleeraufnahme, Abdomenübersichtsaufnahme) ohne Kontrastmittel kann zur Steindiagnostik, zur Feststellung der Röntgendichte und zur Nachkontrolle bei röntgendichten Konkrementen hilfreich sein.

Gesamtabstimmung: $96 \%$

\section{Computertomographie}

Verglichen mit der axialen Schichtung helfen koronare Rekonstruktionen der CT die maximale Größe bei Uretersteinen besser einzuschätzen. Diekraniokaudale Ausdehnung von Steinen wird bei der axialen Ansicht, wahrscheinlich aufgrund des Partial-volume-Effekts, deutlich überschätzt, verglichen mit der koronaren Ansicht. Sekundärpathologien und Zufallsbefunde können in $>10 \%$ der nativen CT gefunden werden [14]. Das Dual-energy-CT ohne Kontrastmittelgabe eignet sich zur In-vivo-Einschätzung der Harnsteinzusammensetzung und einer möglichen Lysetherapie [15-21].

\section{Statement geändert}

Der Einsatz einer CT ohne Kontrastmittel erlaubt eine Einschätzung der Steindichte (in Hounsfield-Units) und anderer Eigenschaften des Steins (Heterogenität, Impaktierung). Gesamtabstimmung: $88 \%$

\section{Low-dose-Computertomographie}

Trotz Einführung von „Low-dose-Protokollen“ für Patienten mit einem Body Mass Index (BMI) $\leq 30$ ist die Strahlendosis im Vergleich zum i.v.-Urogramm und der Abdomenübersichtsaufnahme erhöht (Low-dose-CT: 0,97-1,9 mSv; reguläre Nativ-CT: 4,5-5 mSv; kontrastverstärktes CT: 25-35 mSv). In der täglichen Routine muss davon ausgegangen werden, dass die Verwendung von Lowdose-Protokollen seltener ist und die mediane effektive Dosis der CT-Untersuchung höher liegt (7,6\% Low-dose-, effektive mediane Dosis $11 \mathrm{mSv}$ ).

Hinsichtlich der Harnsteindetektion zeigen sich keine Unterschiede zwischen "Low-dose-“ und konventioneller nativer CT bei einer Reduktion der effektiven Dosis um 1/4 in der Low-dose-CT $[22,23]$. Die digitale Tomosynthese stellt mit geringerer Strahlenbelastung, guter Sensitivität und niedrigeren Kosten eine Alternative zum nativen CT dar, ist jedoch nicht flächendeckend verfügbar [24].

\section{Magnetresonanztomographie}

In der Magnetresonanztomographie (MRT) werden Konkremente nicht direkt, sondern lediglich als Füllungsdefekt im Urin abgebildet. Ist eine Strahlenexposition kontraindiziert (z. B. Schwangerschaft), kann bei fehlender Aussagekraft der Sonographie das MRT eine diagnostische Alternative zum Nachweis einer Dilatation darstellen. Dennoch ist das MRT aufgrund der geringen Sensitivität zur Harnsteindiagnostik ungeeignet und kann diese nur als Füllungsdefekt darstellen [25].

\section{Notfalldiagnostik}

Ein Nativ-CT stellt die weiterführende Standarddiagnostik bei Verdacht auf eine Urolithiasis dar. Insbesondere bei Verdacht auf Urosepsis, Fieber oder bei Einzelniere muss eine sofortige Diagnosesicherung und Therapie angestrebt werden. Neuere Untersuchungen zeigen, dass eine Sonographie oder die verzögerte Durchführung einer CT bei geringer Interventionswahrscheinlichkeit Alternativen darstellen und so die Zahl der CT-Untersuchungen verringert werden kann [26].

\section{Präinterventionelle Bildgebung}

Die Art der Bildgebung wird durch die klinische Situation bestimmt und ist u.a. abhängig davon, ob ein Verdacht auf einen Nieren- oder Harnleiterstein vorliegt. Die präinterventionelle Bildgebung kann mittels i.v.-Urographie, kontrastmittelverstärkter CT sowie retro- oder ggf. antegrader Ureteropyelographie in speziellen Situationen (Kinder, Schwangerschaft) mit einer MR-Urographie erreicht werden. Diese liefert Informationen zur Anatomie, kann aber den Stein nur indirekt darstellen.

\section{Empfehlung geändert}

Für die interventionelle Harnsteinbehandlung ist, insbesondere bei Nierensteinen, die Kenntnis der Konfiguration des Hohlsystems erforderlich. Dies erfordert in der Regel eine Kontrastmittelbildgebung.

\section{Gesamtabstimmung: $96 \%$}

\section{Postinterventionelle Bildgebung}

Nach Durchführung einer extrakorporalen Stoßwellenlithotripsie (ESWL), Ureterorenoskopie (URS) oder perkutanen Nephrolitholapaxie (PCNL) dient der Nachweis von Restfragmenten der Indikationsstellung zu einer neuerlichen Therapie. Bei einer Bildgebung innerhalb der ersten Woche nach der Ersttherapie, können falsch-positive Befunde von spontanabgangsfähigen Restfragmenten $\mathrm{zu}$ einer Übertherapie führen. Da es bezüglich des idealen Zeitpunktes einer Kontrollbildgebung oder Indikationsstellung zum „second look“ keine ausreichende Evidenz gibt, bleibt dieser der Einschätzung des behandelnden Arztes überlassen.

\section{Empfehlungen neu}

Bei einer In-toto-Harnsteinextraktion bei der URS oder PCNL kann eine postinterventionelle Bildgebung entfallen. Gesamtabstimmung: $96 \%$

Bei der ESWL bzw. anderen interventionellen Lithotripsietechniken besteht keine Einigkeit über Art und Zeitpunkt der postinterventionellen Bildgebung. 


\section{Gesamtabstimmung: $92 \%$}

\section{Behandlung von Patienten mit Nierenkolik}

\section{Schmerztherapie}

In einem Cochrane Review zeigten sich nichtsteroidale Antiphlogistika, Paracetamol und Pyrazolon-Derivate (Metamizol) Opioiden in der Behandlung der akuten Nierenkolik überlegen [27]. Beachtenswert ist bei Diclofenac und Ibuprofen ein erhöhtes Risiko kardialer ischämischer Ereignisse bei Gefäßpatienten. Diese sollten daher nur unter sorgfältiger Abwägung gegeben werden. Diclofenac ist u. a. kontraindiziert bei Patienten mit schwerer Niereninsuffizienz, kongestiver Herzinsuffizienz, ischämischer Herzkrankheit und peripherer arterieller und zerebrovaskulärer Erkrankung.

\section{Empfehlung neu}

Zur Therapie akuter steinbedingter Schmerzen („Kolik“) sind Metamizol, Paracetamol und Diclofenac (bei normaler Nierenfunktion) wegen der höheren Effektivität und geringerer Nebenwirkungen Opioiden vorzuziehen. Gesamtabstimmung: $79 \%$

\section{Empfehlungen neu}

Bei persistierenden Beschwerden (,Status colicus") soll die Einlage einer Harnleiterschiene oder perkutanen Nephrostomie zur Dekompression oder die primäre Steinentfernung angeboten werden.

Gesamtabstimmung: $100 \%$

Die stationäre Behandlung ist bei Patienten, die sich notfallmäßig mit kolikartigen Flankenschmerzen in einem Krankenhaus vorstellen, bis zur Diagnosestellung und Festlegung eines vorläufigen Therapieplans gerechtfertigt.

Bei MET („medical expulsive therapy“) muss vor Entlassung zumindest eine Infektion ausgeschlossen und eine ausreichende Analgesie erreicht sein. Dabei ist die Kooperationsfähigkeit des Pati-

Urologe 2019.58:1304-1312 https://doi.org/10.1007/s00120-019-01033-7

(c) Der/die Autor(en) 2019

C. Seitz - T. Bach • M. Bader - W. Berg · T. Knoll • A. Neisius · C. Netsch • M. Nothacker •

S. Schmidt · M. Schönthaler · R. Siener · R. Stein · M. Straub - W. Strohmaier · C. Türk · B. Volkmer

\section{Aktualisierung der S2k-Leitlinie zur Diagnostik, Therapie und Metaphylaxe der Urolithiasis (AWMF Registernummer 043-025). Was ist neu?}

Zusammenfassung

Die Zunahme des medizinischen Wissens, technische Neuerungen gemeinsam mit demographischem Wandel stellen eine Herausforderung an die Neukonzeption von Leitlinien und klinischen Studien dar. Die vorliegende S2k-Leitlinie, die sich ausschließlich mit Nieren- und Harnleitersteinen beschäftigt, soll die Behandlung von Harnsteinpatienten in Klinik und Praxis unterstützen, aber auch Patienteninformationen zur Urolithiasis geben. Die zunehmende interdisziplinäre Zusammenarbeit in der Steintherapie zeigt sich auch an der Anzahl beteiligter Fachgruppen und Arbeitsgemeinschaften in der Erstellung des neuen Leitlinienupdates. Die vorliegende, aus einem interdisziplinären Konsensusprozess hervorgegangene S2k-Leitlinie stellt die aktuellen Empfehlungen praxisnah dar und gibt Entscheidungshilfen für Diagnostik-, Therapie- und Metaphylaxemaßnahmen auf Basis von Expertenmeinungen und verfügbaren Evidenzgrundlagen aus der Literatur.

Schlüsselwörter

Stoßwellenlithotripsie, extrakorporale . Ureterorenoskopie $\cdot$ Nephrolitholapaxie, perkutane $\cdot$ Harnsteinbildner $\cdot$ Hyperparathyreoidismus

Update of the 2Sk guidelines on the diagnostics, treatment and metaphylaxis of urolithiasis (AWMF register number 043-025). What is new?

Abstract

The increase of medical knowledge and technical innovations together with the demographic change represent a challenge for the new conception of guidelines and clinical studies. The present S2k guidelines, which are exclusively concerned with kidney and ureteral stones, should support the treatment of urolithiasis in hospitals and private practices and provide information on urolithiasis for patients. Increasing interdisciplinary collaboration in stone treatment is also demonstrated in the number of professional and working groups participating in the update of the new guidelines. The present S2k guidelines emerged from a consensus process and demonstrate the current recommendations in step with actual practice. They provide decision-making guidance for diagnostics, treatment and metaphylactic measures based on expert opinions and available published fundamental evidence from the literature.

Keywords

Extracorporal shock-wave lithotripsy - Ureterorenoscopy - Nephrolithotomy, percutaneous . Urinary calculi $\cdot$ Hyperparathyroidism enten zu berücksichtigen.

\section{Konservative Therapie von Harnleitersteinen}

Mit der verfügbaren Evidenz lässt sich nicht feststellen, bis zu welcher Steingröße ein Spontanabgang zu erwarten ist oder mit welchen Symptomen er einhergehen wird. Jedenfalls nimmt mit zunehmender Steingröße und proximaler Lage die Wahrscheinlichkeit eines Spontanabgangs ab (• Tab. 1).

\section{Empfehlung geändert}

Bei Patienten mit neu diagnostiziertem Harnleiterstein bis $7 \mathrm{~mm}$ kann der Spontanabgang unter regelmäßiger Kontrolle abgewartet werden. Gesamtabstimmung: $79 \%$ 
Tab. 1 Wahrscheinlichkeit des spontanen Uretersteinabgangs [28, 29]

\begin{tabular}{|l|l|l|l|}
\hline & Steingröße & $\begin{array}{l}\text { Anteil spontan abgegangener } \\
\text { Steine (\%) } \\
(\mathbf{9 5} \%-\mathbf{K I})\end{array}$ & $\begin{array}{l}\text { Dauer bis zum Steinab- } \\
\text { gang (Tage) }\end{array}$ \\
\hline $\begin{array}{l}\text { Preminger } \\
\text { et al. [28] }\end{array}$ & $\begin{array}{l}<5 \mathrm{~mm} \\
(n=224) \\
5-10 \mathrm{~mm} \\
(n=104)\end{array}$ & $68(46-85)$ & - \\
\hline $\begin{array}{l}\text { Ye et al. } \\
{[29]}\end{array}$ & $\begin{array}{l}4-5 \mathrm{~mm} \\
(n=1654)\end{array}$ & 87 & $6,1 \pm 3,2$ \\
\hline & $\begin{array}{l}6-7 \mathrm{~mm} \\
(n=1093)\end{array}$ & 75 & $12,5 \pm 3,3$ \\
\hline KI Konfidenzintervall & & \\
\hline
\end{tabular}

\section{Medikamentöse Supportiv- medikation}

Mehrere Metaanalysen, darunter ein Cochrane Review, bestätigen die Wirksamkeit einer supportiven medikamentösen Therapie (MET), insbesondere bei Uretersteinen $>5 \mathrm{~mm}$. Diese erhöht die Wahrscheinlichkeit eines spontanen Steinabgangs und verringert die Zeit und den Schmerzmittelbedarf bis zum Steinabgang [30]. Dabei wird bei zunehmendem Steindurchmesser und proximaler Lage im Harnleiter eine Spontanausscheidung unwahrscheinlicher. Pickard et al. [31] konnten in einer multizentrischen, randomisierten Studie zwar keinen Effekt der MET auf die Steinausscheidungsrate zeigen, die Studie war allerdings auch nicht für Steingrößen $>5 \mathrm{~mm}$ gepowert. Der Endpunkt war eine fehlende Interventionsbedürftigkeit 4 Wochen nach Randomisierung und nicht, wie in der bisher größten randomisierten Studie von Ye et al. [29], die CT-basierte Steinfreiheitsrate distaler Uretersteine.

\section{Medikamente}

Verbesserte spontane Ausscheidungsraten durch MET wurden für $a$-Blocker (u. a. Tamsulosin) und den Kalziumantagonisten Nifedipin nachgewiesen [30]. Im randomisierten Vergleich wurden für Tamsulosin und andere $\alpha$-Blocker vergleichbare Effekte gezeigt.

\section{Einflussgrößen (Steingröße/ Lokalisation)/Dauer der Behandlung}

Die bisher größte multizentrische, randomisierte, doppelt verblindete Studie von Ye et al. [29] konnte an über 3400 Patienten einen signifikanten Vorteil in der Ausscheidungsrate nach Tamsulosin-Gabe gegenüber Placebo bei distalen Uretersteinen $>5$ bis $7 \mathrm{~mm}$ ( 87 vs. $75 \%$ ) zeigen.

Eine 55 Publikationen umfassende Metaanalyse untersuchte als primären Endpunkt die spontane Steinpassage und konnte zeigen, dass $a$-Blocker den spontanen Abgang großer Konkremente in allen Abschnitten des Harnleiters fördern [32]. Zur Behandlungsdauer gibt es keine exakten Untersuchungen. In einer Metaanalyse wurden in den einbezogenen Studien eine mittlere Dauer bis zum Steinabgang zwischen 1 und 12 Tagen angegeben, der Beobachtungszeitraum lag zwischen 3 und 6 Wochen.

\section{„Off-label-Gebrauch"}

Patienten, die eine MET durchführen, sind über eine Off-label-Anwendung zu informieren. Für alle Medikamentengruppen gilt, dass Patienten auf den Offlabel-Gebrauch und mögliche Nebenwirkungen wie retrograde Ejakulation oder Hypotonie vor Therapiebeginn hinzuweisen sind. Des Weiteren sind entsprechende Kontraindikationen (bekannte orthostatische Hypotonie, schwere Leberinsuffizienz, schwere Herzerkrankung) zu beachten.

Für Tamsulosin hat der Gemeinsame Bundesausschuss im März 2019 ei- ne Änderung der Arzneimittelrichtlinie herausgegeben [33]. Hierin sind die verordnungsfähigen Arzneimittel genannt, welche eine Zustimmung der pharmazeutischen Unternehmen für die „Offlabel-Anwendung" erhalten haben.

\section{Statement geprüft}

Die medikamentöse expulsive Therapie (MET) kann die Steinabgangsrate nach Lithotripsie durch ESWL und URS signifikant beschleunigen, die Steinfreiheitsraten erhöhen und den Schmerzmittelbedarf senken.

Gesamtabstimmung: $100 \%$

\section{Kinderurologie}

Je früher der Beginn der Steinerkrankung im Kindesalter auftritt, umso größer ist die Wahrscheinlichkeit, dass eine metabolische Erkrankung die Nierensteinproblematik auslöst. Demzufolge sollte sie auch schnell diagnostiziert werden sollte um im Langzeitverlauf teils drastische Probleme zu vermeiden (z.B. frühzeitiges Nierenversagen bei primärer Hyperoxalurie Typ I). Die Inzidenz der Blasensteine hat in den entwickelten Ländern deutlich abgenommen, ist aber in den Entwicklungsländern aufgrund der Ernährungssituation weiterhin ein Problem [34].

\section{Klinische Symptomatik}

Bei kleineren Kindern findet man oft nur Symptome der Gereiztheit und unspezifische Bauchschmerzen in der Nabelgegend, auch mit Erbrechen [35]. Bei vielen betroffenen Kindern sind die einzigen Hinweise lediglich eine Mikrohämaturie oder eine Harnwegsinfektion, wobei die alleinige asymptomatische, idiopathische Mikrohämaturie selten auf eine Steinprädisposition hinweist.

\section{Bildgebung bei Kindern}

\section{Empfehlungen geprüft}

Die Ultraschalldiagnostik soll bei Kindern als Verfahren der ersten Wahl erfolgen. 
Tab. 2 Eine Tabelle mit Darstellung der Hochrisikogruppen der Harnsteinbildner wurde neu

\section{erstellt}

\begin{tabular}{|c|c|}
\hline \multirow{6}{*}{$\begin{array}{l}\text { Allgemeine } \\
\text { Faktoren }\end{array}$} & Frühes Auftreten von Urolithiasis (insbesondere Kinder und Jugendliche) \\
\hline & Familiäre Steinformation \\
\hline & Brushit-haltige Steine $\left(\mathrm{CaHPO}_{4} \times 2 \mathrm{H}_{2} \mathrm{O}\right)$ \\
\hline & Harnsäure und harnsäurehaltige Steine \\
\hline & Infektsteine \\
\hline & $\begin{array}{l}\text { Einzelniere (die Niere selbst erhöht das Risiko der Steinbildung nicht be- } \\
\text { sonders, aber die Verhinderung des Wiederauftretens von Steinen ist von } \\
\text { größerer Bedeutung) }\end{array}$ \\
\hline \multirow{7}{*}{$\begin{array}{l}\text { Mit der } \\
\text { Steinbildung } \\
\text { assoziierte } \\
\text { Erkrankungen }\end{array}$} & Hyperparathyreoidismus \\
\hline & Metabolisches Syndrom \\
\hline & Nephrokalzinose \\
\hline & Polyzystische Nierenerkrankung (PKD) \\
\hline & $\begin{array}{l}\text { Chronische Magen-Darm-Erkrankungen (z. B. Jejunoilealbypass, Darmresek- } \\
\text { tion, Morbus Crohn, malabsorptive Erkrankungen, enterische Hyperoxalurie } \\
\text { nach Harnableitung) und bariatrische Chirurgie }\end{array}$ \\
\hline & Sarkoidose \\
\hline & Rückenmarksverletzung, neurogene Blase \\
\hline \multirow{8}{*}{$\begin{array}{l}\text { Genetisch } \\
\text { bestimmte } \\
\text { Steinbildung }\end{array}$} & Zystinurie (Typ A, B und AB) \\
\hline & Primäre Hyperoxalurie $(\mathrm{PH})$ \\
\hline & Renale tubuläre Azidose (RTA) Typ I \\
\hline & 2,8-Dihydroxyadeninurie \\
\hline & Xanthinurie \\
\hline & Lesch-Nyhan-Syndrom \\
\hline & Mukoviszidose \\
\hline & ArzneimittelinduzierteSteinbildung \\
\hline \multirow{7}{*}{$\begin{array}{l}\text { Anatomische } \\
\text { Anomalien bei } \\
\text { der Steinbildung }\end{array}$} & Markschwammniere (tubuläre Ektasie) \\
\hline & Subpelvine Harnleiterstenose (UPJ) \\
\hline & Kelchdivertikel, Kelchzyste \\
\hline & Ureterstriktur \\
\hline & Vesikoureterorenaler Reflux \\
\hline & Hufeisenniere \\
\hline & Ureterozele \\
\hline \multirow[t]{2}{*}{ Umweltfaktoren } & Chronische Bleibelastung \\
\hline & Cadmium \\
\hline
\end{tabular}

Gesamtabstimmung: $100 \%$

Empfehlung geändert

Bei unklaren Fällen kann bei Kindern eine native Low-dose-CT durchgeführt werden.

Gesamtabstimmung: $88 \%$

\section{Empfehlung geändert}

Zur Therapieplanung sollte bei Kindern, soweit möglich, eine MR-Urographie aus strahlenhygienischen Gründen dem Ausscheidungsurogramm vorgezogen werden.

\section{Gesamtabstimmung: $88 \%$}

\section{Interventionelle Therapie}

Eine Therapie, d.h. eine Steinentfernung, ist nur bei symptomatischen Steinen, bei Ausgusssteinen oder bei Infektsteinen zielgerichtet $\mathrm{zu}$ planen und sollte bei Kindern immer nur in erfahrenen Zentren mit kindgerechter Ausstattung und kleinkalibrigen Instrumenten durchgeführt werden. Bei kleinen asymptomatischen Patienten soll der Stein nicht unbedingt entfernt werden. Vielmehr sollte in dieser Situation primär eine Stoffwechselabklärung erfolgen.

\section{MET bei Kindern}

Diese Standardtherapie bei Erwachsenen hat bei Kindern bisher eine geringe Evidenz, obwohl die Wirksamkeit der $\alpha$-Blocker, insbesondere Tamsulosin, beschrieben ist. Eine Metaanalyse von fünf randomisierten Studien zeigte, dass Tamsulosin effektiver zu sein scheint als Doxazosin [36].

\section{Metabolische Abklärung und Rezidivprophylaxe}

\section{Empfehlung geändert}

Jedes Kind mit einem ersten Harnstein soll einer erweiterten metabolischen Abklärung zugeführt werden. Gesamtabstimmung: $100 \%$ Es wurden neue Tabellen für die Oxalatund Glykolatkonzentrationen im Plasma sowie 24-h-Sammelurinwerte bei Kindern aller Altersgruppen erstellt sowie molare Kreatininquotienten verschiedener Altersgruppen (von 0 Monaten bis über 14 Jahre).

Gesamtabstimmung: $96 \%$

\section{Metabolische Diagnostik und Therapie}

\section{Basisdiagnostik}

Grundlage der Einteilung in Niedrig- und Hochrisikogruppe ist die Harnsteinanalyse sowie die Basisdiagnostik. Patienten der Niedrigrisikogruppe bedürfen keiner weiteren Abklärung, für sie sind die Maßnahmen der „Allgemeinen Harnsteinmetaphylaxe" ausreichend. Demgegenüber ist bei Patienten der Hochrisikogruppe eine erweiterte, steinartspezifische metabolische Abklärung sinnvoll, um anhand des ermittelten biochemischen Risikoprofils anschließend eine gezielte pharmakologische Metaphylaxe einzuleiten.

\section{Empfehlung geändert}

Jeder Steinpatient sollte entsprechend seinem Rezidivrisiko der Niedrig- oder Hochrisikogruppe zugeordnet werden (- Tab. 2). 
Gesamtabstimmung: $100 \%$

\section{Steinanalyse}

Die bisherige Empfehlung eine Steinanalyse bei jeder neuen Steinepisode durchzuführen wurde präzisiert:

\section{Empfehlung geändert}

Im Rezidivfall ist eine erneute Steinanalyse erforderlich bei:

- Wiederholung unter pharmakologischer Prävention;

- frühes Wiederauftreten nach interventioneller Therapie mit vollständiger Steinsanierung;

- spätes Wiederauftreten nach einer längeren steinfreien Zeit.

\section{Gesamtabstimmung: $96 \%$}

\section{Erweiterte metabolische} Diagnostik

\section{Empfehlung geändert}

Zur erweiterten metabolischen Diagnostik gehört neben einer Blutuntersuchung die Auswertung zweier korrekt gewonnener 24-h-Sammelurine. Gesamtabstimmung: $96 \%$

\section{Steinartspezifische Rezidiv- prophylaxe (spezifische Metaphylaxe)}

Die Maßnahmen der allgemeinen Harnsteinmetaphylaxe gelten als Basistherapie, die - in Abhängigkeit vom individuellen Risikoprofil eines Patienten - durch eine steinartspezifische, ernährungsmedizinische und pharmakologische Therapie ergänzt werden sollte. Eine spezifische Rezidivprophylaxe (Metaphylaxe) ist nur für Patienten der Hochrisikogruppe erforderlich.

\section{Befundinterpretation und ätiologische Grundlagen}

Risikofaktoren der Kalziumoxalatsteinbildung sind ein primärer und sekundärer Hyperparathyreoidismus, eine primäre Hyperoxalurie, eine renal-tubuläre Azidose sowie ein Fettmalab- sorptionssyndrom, wie sie bei chronisch entzündlichen Darmerkrankungen oder nach Darmchirurgie auftreten können. Allerdings finden sich bei ca. $70 \%$ der betroffenen Patienten keiner dieser Risikofaktoren. Man spricht dann von idiopathischen Kalziumoxalatsteinbildnern. Es sollten unbedingt auch Stoffwechselerkrankungen, die entweder zur alleinigen Hyperkalziurie oder aber zur simultanen Hyperkalzämie und Hyperkalziurie führen mit in der Differentialdiagnostik bedacht werden (z.B. Williams-Beuren-Syndrom, „Dent's disease“, BartterSyndrom u.a.m.).

\section{Rezidivprophylaxe (Metaphylaxe) - pharmakologisch}

\section{Zystin-Steine}

Tiopronin gilt als Mittel der ersten Wahl zur Disulfidbrückenspaltung. Die Evidenzlage bezüglich des ACE-Hemmers (,angiotensin-converting enzyme“) Captopril und Vitamin C (Ascorbinsäure), die zu einer Senkung der Zystin-Konzentration im Urin durch Spaltung der Disulfidbrücke führen sollen, ist für eine Empfehlung als nicht mehr ausreichend anzusehen.

\section{Hydrochlorothiazid}

Aufgrund neuester Daten besteht bei Patienten mit Langzeitanwendung von Hydrochlorothiazid ein erhöhtes vorkommen des weißen Hautkrebses (spinozelluläres Karzinom). Es wird daher bis zum Vorliegen weiterer Ergebnisse empfohlen für die Korrektur der Hyperkalziurie auf Chlorthalidon oder Indapamid auszuweichen [37-39].

\section{Hyperurikosurie}

Die Hyperurikosurie begünstigt ein Kalziumoxalatkristallwachstum, weil es zwischen Harnsäure- und Kalziumoxalatkristallen zur Kokristallisation kommt. Eine Senkung der Harnsäure im Urin hat also auch bei Kalziumoxalatsteinen einen rezidivprophylaktischen Nutzen. Allopurinol senkt nachweislich in einer Dosierung von 100-300 mg bei hyperurikosurischen Kalziumoxalatsteinbildnern die Steinrezidivrate. Als Alternative zu Allopurinol steht Febuxostat zur Verfügung, allerdings muss man bei dieser Substanz ein verschärftes Nebenwirkungsprofil beachten.

\section{Stoffwechselstörungen}

und Erkrankungen, die zur

Kalziumsteinbildung führen

\section{Primärer Hyperparathyreoidismus}

Ein erhöhtes ionisiertes Kalzium in der Basisdiagnostik kann auf einen primären Hyperparathyreoidismus (pHPT) hinweisen und zieht die Bestimmung des Parathormons im Serum nach sich. Sprechen beide Laborparameter für das Vorliegen eines pHPT, erfolgt eine Ultraschall- oder Schnittbilddiagnostik der Halsregion zur Bestätigung eines Nebenschilddrüsenadenoms. Die Therapie besteht klassischerweise in der chirurgischen Resektion des Nebenschilddrüsenadenoms. Seit kurzer Zeit steht mit Cinacalcet auch ein medikamentöser Ansatz als vorübergehende Therapiemöglichkeit zur Verfügung [40].

\section{Harnsäuresteine}

\section{Befundinterpretation und ätiologische Grundlagen}

Die Hyperurikosurie kann exogen bedingt auf die (fehlerhafte) Ernährung zurückzuführen sein. Es gibt auch endogene Ursachen wie Gicht, Enzymdefekte, myeloproliferative Störungen, Tumorlysesyndrom, Medikamente oder katabole Stoffwechsellagen.

Ein niedriger $\mathrm{pH}$-Wert im Urin kann verursacht sein durch: eine verminderte Ammoniumausscheidung im Urin (Insulinresistenz), eine erhöhte endogene Säureproduktion (Insulinresistenz, metabolisches Syndrom oder durch Bewegung induzierte Laktatazidose), eine erhöhte Säurezufuhr (hohe tierische Proteinzufuhr) oder einen vermehrten Basenverlust (Durchfall).

\section{Fazit für die Praxis}

- Zur Therapie akuter steinbedingter Schmerzen („Kolik“) sind Metamizol, Paracetamol und Diclofenac (bei normaler Nierenfunktion) wegen der höheren Effektivität und geringerer Nebenwirkungen Opioiden vorzuziehen. 
- Bei Patienten mit neu diagnostiziertem Harnleiterstein bis $7 \mathrm{~mm}$ kann der Spontanabgang unter regelmäßiger Kontrolle abgewartet werden.

- Bei Kindern erfolgt die primäre Bildgebung mittels Ultraschall. Ist diese nicht eindeutig, kann eine native Low-dose-CT durchgeführt werden. Die Abdomenübersichtsaufnahme ist entbehrlich.

- Wenn zur Therapieplanung die Darstellung des Hohlraumsystems bei Kindern notwendig ist, dann sollte die Magnetresonanzurographie dem Ausscheidungsurogramm vorgezogen werden.

- Kleinere asymptomatische Steine bei Kindern bedürfen nicht unbedingt einer aktiven Therapie, eine metabolische Abklärung sollte in jedem Fall erfolgen.

- Die stationäre Behandlung ist bei Patienten, die sich notfallmäßig mit kolikartigen Flankenschmerzen in einem Krankenhaus vorstellen, bis zur Diagnosestellung und Festlegung eines vorläufigen Therapieplans gerechtfertigt.

\section{Korrespondenzadresse}

\section{Prof. Dr. C. Seitz}

Urologische Universitätsklinik, Medizinische Universität Wien, Allgemeines Krankenhaus der Stadt Wien

Währinger Gürtel 18-20, 1090 Wien, Österreich drseitz@gmx.at

Funding. Open access funding provided by Medical University of Vienna.

\section{Einhaltung ethischer Richtlinien}

Interessenkonflikt. C. Seitz, T. Bach, M. Bader, W. Berg, T. Knoll, A. Neisius, C. Netsch, M. Nothacker, S. Schmidt, M. Schönthaler, R. Siener, R. Stein, M. Straub, W. Strohmaier, C. Türk und B. Volkmer geben an, dass kein Interessenkonflikt besteht.

Für diesen Beitrag wurden von den Autoren keine Studien an Menschen oder Tieren durchgeführt. Für die aufgeführten Studien gelten die jeweils dort angegebenen ethischen Richtlinien.

Open Access Dieser Artikel wird unter der Creative Commons Namensnennung 4.0 International Lizenz (http://creativecommons.org/licenses/by/4.0/deed. de) veröffentlicht, welche die Nutzung, Vervielfältigung, Bearbeitung, Verbreitung und Wiedergabe in jeglichem Medium und Format erlaubt, sofern Sie den/die ursprünglichen Autor(en) und die Quelle ordnungsgemäßnennen, einen Linkzur Creative Commons Lizenz beifügen und angeben, ob Änderungen vorgenommen wurden.

\section{Literatur}

1. Chen TT, Wang C, Ferrandino MN, Scales CD, Yoshizumi TT, Preminger GM et al (2015) Radiation exposure during the evaluation and management of nephrolithiasis. JUrol 194(4):878-885

2. Weisenthal K, Karthik P, Shaw M, Sengupta D, Bhargavan-Chatfield M, Burleson J et al (2018) Evaluation of kidney stones with reduced-radiation dose CT: progress from 2011-2012 to 2015-2016-not there yet. Radiology 286(2):581-589

3. Tonolini M, Valconi E, Vanzulli A, Bianco R (2018) Radiation overexposure from repeated $\mathrm{CT}$ scans in young adults with acute abdominal pain. Emerg Radiol 25(1):21-27

4. MillsL, MorleyEJ, SoucyZ, VilkeGM,LamSHF (2018) Ultrasound for the diagnosis and management of suspected Urolithiasis in the emergency department. JEmerg Med 54(2):215-220

5. Sade R, Ogul H, Eren S, Levent A, Kantarci M (2017) Comparison of ultrasonography and low-dose computed tomography for the diagnosis of pediatric urolithiasis in the emergency department Eurasian J Med 49(2):128-131

6. Metzler IS, Smith-Bindman R, Moghadassi M, Wang RC, Stoller ML, Chi T (2017) Emergency department imaging modality effect on surgical management of nephrolithiasis: a multicenter, randomized clinical trial. JUrol 197(3 Pt 1):710-714

7. Park YH, Jung RB, Lee YG, Hong CK, Ahn JH, Shin TY et al (2016) Does the use of bedside ultrasonography reduce emergency department length of stay for patients with renal colic?: a pilot study. Clin ExpEmerg Med 3(4):197-203

8. Abdel-Gawad M, Kadasne RD, Elsobky E, Ali-ElDein B, Monga M (2016) A prospective comparative study of color doppler ultrasound with twinkling and noncontrast computerized tomography for the evaluation of acute renal colic. J Urol 196(3):757-762

9. Sen V, Imamoglu C, Kucukturkmen I, Degirmenci T, Bozkurt IH, Yonguc T et al (2017) Can Doppler ultrasonography twinkling artifact be used as an alternative imaging modality to non-contrastenhanced computed tomography in patients with ureteral stones? A prospective clinical study. Urolithiasis 45(2):215-219

10. Dai JC, Dunmire B, Sternberg KM, Liu Z, Larson T, Thiel J et al (2018) Retrospective comparison of measured stone size and posterior acoustic shadow width in clinical ultrasound images. World JUrol 36(5):727-732

11. Ganesan V, De S, Greene D, Torricelli FC, Monga M (2017) Accuracy of ultrasonography for renal stone detection and size determination: is it good enough for management decisions? BJU Int 119(3):464-469

12. Dunmire B, Lee FC, Hsi RS, Cunitz BW, Paun M, Bailey MRetal (2015) Tools to improve the accuracy of kidney stone sizing with ultrasound. J Endourol 29(2):147-152

13. Dunmire B, Harper JD, Cunitz BW, Lee FC, Hsi R, Liu Z et al (2016) Use of the acoustic shadow width to determine kidney stone size with ultrasound. JUrol 195(1):171-177

14. Samim M, Goss S, Luty S, Weinreb J, Moore C (2015) Incidental findings on CT for suspected renal colic in emergency department patients: prevalence and types in 5,383 consecutive examinations. J Am Coll Radiol 12(1):63-69

15. Franken A, Gevenois PA, Muylem AV, Howarth N, Keyzer C (2018) In vivo differentiation of uric acid versus non-uric acid urinary calculi with third-generation dual-source dual-energy $C T$ at reduced radiation dose. AJR Am J Roentgenol 210(2):358-363

16. Lombardo F, Bonatti M, Zamboni GA, Avesani G, Oberhofer N, Bonelli Met al (2017) Uric acid versus non-uric acid renal stones: in vivo differentiation with spectral CT. Clin Radiol 72(6):490-496

17. Chaytor RJ, Rajbabu K, Jones PA, McKnight L (2016) Determining the composition of urinary tract calculi using stone-targeted dual-energy CT: evaluation of a low-dose scanning protocol in a clinical environment. Br JRadiol 89(1067):20160408

18. Zhang GM, Sun H, Xue HD, Xiao H, Zhang XB, Jin ZY (2016) Prospective prediction of the major component of urinary stone composition with dual-source dual-energy CT in vivo. Clin Radiol 71(11):1178-1183

19. Wilhelm K, Schoenthaler M, Hein S, Adams F, Schlager D, Kuehhas FE et al (2015) Focused dualenergy CTmaintains diagnosticand compositional accuracy for urolithiasis using ultralow-dose noncontrast CT. Urology 86(6):1097-1102

20. Zheng X, Liu Y, Li M, Wang Q, Song B (2016) Dualenergy computed tomography for characterizing urinary calcified calculi and uric acid calculi: a meta-analysis. Eur J Radiol 85(10):1843-1848

21. Akand M, Koplay M, Islamoglu N, Gul M, Kilic O, Erdogdu MB (2016) Role of dualsource dual-energy computed tomography versus X-ray crystallography in prediction of the stone composition: a retrospective non-randomized pilot study. Int Urol Nephrol 48(9):1413-1420

22. Moore CL, Daniels B, Singh D, Luty S, Gunabushanam G, Ghita M et al (2016) Ureteral stones: implementation of a reduced-dose $\mathrm{CT}$ protocol in patients in the emergency department with moderate to high likelihood of calculi on the basis of STONE score. Radiology 280(3):743-751

23. Xiang H, Chan M, Brown V, Huo YR, Chan L, Ridley L (2017) Systematic review and meta-analysis of the diagnostic accuracy of low-dose computed tomography of the kidneys, ureters and bladder for urolithiasis. J Med Imaging Radiat Oncol 61(5):582-590

24. Cabrera FJ, Kaplan AG, Youssef RF, Tsivian M, Shin RH, Scales CD et al (2016) Digital tomosynthesis: a viable alternative to noncontrast computed tomography for the follow-up of nephrolithiasis? JEndourol 30(4):366-370

25. Ibrahim EH, Cernigliaro JG, Bridges MD, Pooley RA, Haley WE (2016) The capabilities and limitations of clinical magnetic resonance imaging for detecting kidney stones: a retrospective study. Int J Biomed Imaging 2016:4935656

26. Hasani SA, Fathi M, Daadpey M, Zare MA, Tavakoli N Abbasi S (2015) Accuracy of bedside emergency physician performed ultrasound in diagnosing different causes of acute abdominal pain: a prospective study. Clin Imaging 39(3):476-479

27. AfsharK, Jafari S, Marks AJ, Eftekhari A, MacNeily AE (2015) Nonsteroidal anti-inflammatory drugs (NSAIDs) and non-opioids for acute renal colic. Cochrane Database Syst Rev. https://doi.org/10. 1002/14651858.CD006027.pub2

28. Preminger GM, Tiselius HG, Assimos DG, Alken P, Buck AC, Gallucci M et al (2007) 2007 Guideline for the management of ureteral calculi. Eur Urol 52(6):1610-1631 


\section{Leitthema}

29. Ye Z, Zeng G, Yang $H$, Tang $K$, Zhang $X$, Li H et al (2018) Efficacy and safety of tamsulosin in medical expulsive therapy for distal ureteral stones with renal colic: a multicenter, randomized, double-blind, placebo-controlled trial. Eur Urol 73(3):385-391. https://doi.org/10.1016/j.eururo. 2017.10.033

30. Campschroer T, Zhu X, Vernooij RWM, Lock TMTW (2018) a-blockers as medical expulsive therapy for ureteric stones: a Cochrane systematic review. BJU Int 122(6):932-945

31. Pickard R, Starr K, MacLennan G, Lam T, Thomas R, Burr J et al (2015) Medical expulsive therapy in adults with ureteric colic: a multicentre, randomised, placebo-controlled trial. Lancet 386(9991):341-349

32. Hollingsworth JM, Canales BK, Rogers MA, Sukumar S, Yan P, Kuntz GM et al (2016) Alpha blockers for treatment of ureteric stones: systematic review and meta-analysis. BMJ 355:i6112

33. G_BA (2019) Beschluss des Gemeinsamen Bundesausschusses über eine Änderung der ArzneimittelRichtlinie (AM-RL): Anlage VI-Off-Label_Use Tamsulosin bei Urolithiasis (als medikamentöse expulsive Therapie auch nach Lithotripsie). B Anz 16.06. ATB1

34. Soliman NA, RizviSAH (2017) Endemic bladder calculi in children. Pediatr Nephrol 32(9):1489-1499

35. Chu DI, Tasian GE, Copelovitch L (2016) Pediatric kidney stones-avoidance and treatment. Curr Treat Options Pediatr 2(2):104-111

36. Tian D, Li N, Huang W, Zong H, Zhang Y (2017) The efficacy and safety of adrenergic alphaantagonists in treatment of distal ureteral stones in pediatric patients: A systematic review and metaanalysis. J Pediatr Surg 52(2):360-365

37. Pedersen SA, Gaist $D$, Schmidt SAJ, Hölmich LR Friis S, Pottegård A (2018) Hydrochlorothiazide use and risk of nonmelanoma skin cancer: a nationwide case-control study from Denmark. J Am Acad Dermatol 78(4):673-681.e9

38. Pottegård $A$, Hallas J, Olesen $M$, Svendsen MT, Habel LA, Friedman GD et al (2017) Hydrochlorothiazide use is strongly associated with risk of lip cancer.J Intern Med 282(4):322-331

39. Gandini S, Palli D, Spadola G, Bendinelli B, Cocorocchio E, Stanganelli I et al (2018) Antihypertensive drugs and skin cancer risk: a review of the literature and meta-analysis. Crit Rev Oncol Hematol 122:1-9

40. Brardi S, Ponchietti R, Duranti E (2016) Nephrolithiasis associated with normocalcemic or hypercalcemic primary hyperparathyroidism: focus on calciomimetics. G Ital Nefrol 33(6). pii: gin/33.6.4.PMID: 28134399

\section{Hier steht eine Anzeige.}

\section{算 Springer}

